\title{
Alteraciones en la visión cromáticas por agentes neurotóxicos en pacientes de 20 a 40 años
}

\author{
Visual chromatic alteration by neurotoxics in patients of $20-40$ age
}

\section{Alteracao visual cromática por neurotóxicos em pacientes de 20 a 40 anos}

\author{
Gabriela Proaño Mosquera \\ gapm2186@gmail.com \\ ORCID: 0000-0002-1761-870X
}

\author{
Mariana de Jesús Arteaga Segarra \\ marianart1993@hotmail.com \\ ORCID: 0000-0003-3966-3840
}

\author{
Instituto Tecnológico Universitario Cordillera, Quito-Ecuador \\ Recibido 14 de octubre 2020 | Arbitrado y aceptado 30 de noviembre 2020 | Publicado en 04 de enero 2021
}

\begin{abstract}
RESUMEN
El estudio busca determinar las alteraciones de la visión cromática por gentes neurotóxicos en pacientes de 20 a 40 años en la parroquia Checa - Ecuador y cuál de ellas genera mayor vulnerabilidad en las células nerviosas fotorreceptoras de la retina. Materiales y Métodos. Se realizó un estudio evaluativo - descriptivo de pruebas en donde participaron 54 personas de edades entre 20 a 40 años que pertenecen al programa social bachillerato acelerado y se les aplicó la historia clínica de detección para determinar si están dentro de los criterios de inclusión. A los 54 pacientes que cumplieron con el requisito, a parte del examen visual completo, se les aplicó el test de Farnsworth que es la prueba más certera y completa para el diagnóstico de las distintas alteraciones de la visión cromática. Resultados. Al realizar la tabulación de datos, se observa que la alteración cromática más frecuente es la deuteranomalía, y que el agente más neurotóxico para las células fotorreceptoras retinales, es el alcohol, afectando mayormente, a la población masculina de entre 31 a 35 años, el segundo neurotóxico más agresivo, es el cigarrillo, afectando a la misma población. Conclusiones. Se determina que los agentes neurotóxicos inciden directamente en el daño de fotorreceptores especializados al color, el daño puede ser imperceptible al principio, pero puede afectar al paciente principalmente en el desarrollo laboral, que es cuando el paciente cae en cuenta que está presentando una alteración cromática, la disminución de la exposición a los mismos, puede revertir el daño si la frecuencia no ha sido prolongada.
\end{abstract}

Palabras clave: Deuteranomalía; Farnsworth; fotorreceptor; neurotóxico; retina
GP: Licenciatura en Optometría, Escuela Politécnica Javeriana del Ecuador. Politécnica Javeriana del Ecuador. Docente de la carrera de optometría. Formación integral de entornos de aprendizaje de educación superior. Instituto Superior Tecnológico Cordillera, Ecuador.

MA: Tecnóloga en optometría cursa estudios de licenciatura en optometría. Instituto Superior Tecnológico

\begin{abstract}
The study seeks to determine the alterations of color vision by neurotoxic people in patients aged 20 to 40 years in the Czech parish - Ecuador and which of them generates greater vulnerability in the photoreceptor nerve cells of the retina. Materials and methods. An evaluative-descriptive study of tests was carried out in which 54 people between the ages of 20 and 40 who belong to the accelerated high school social program participated and the clinical history of detection was applied to them to determine if they were within the inclusion criteria. To the 54 patients who fulfilled the requirement, apart from the complete visual examination, the Farnsworth test was applied, which is the most accurate and complete test for the diagnosis of the different color vision alterations. Results. When performing the data tabulation, it is observed that the most frequent chromatic alteration is deuteranomaly, and that the most neurotoxic agent for retinal photoreceptor cells is alcohol, affecting mainly the male population between 31 and 35 years of age. The second most aggressive neurotoxic is cigarettes, affecting the same population. Conclusions. It is determined that neurotoxic agents directly affect the damage of specialized photoreceptors to color, the damage may be imperceptible at first, but it can affect the patient mainly in work development, which is when the patient realizes that he is presenting a chromatic alteration, decreasing the exposure to them, can reverse the damage if the frequency has not been prolonged.
\end{abstract}

Key words: Deuteranomaly; Farnsworth; photoreceptor; neurotoxic; retina 
GP: Licenciatura en Optometría, Escuela Politécnica Javeriana del Ecuador. Docente de la carrera de optometría. Formación integral de entornos de aprendizaje de educación superior. Instituto Superior Tecnológico Cordillera, Ecuador.

MA: Tecnóloga en optometría cursa estudios de licenciatura en optometría. Instituto Superior Tecnológico Cordillera, Ecuador.

\section{RESUMO}

0 estudo visa determinar as alterações da visão de cores por pessoas neurotóxicas em pacientes de 20 a 40 anos na freguesia checa-Equador e qual delas gera maior vulnerabilidade nas células nervosas fotorreceptoras da retina. Materiais e métodos. Foi realizado um estudo descritivo-avaliativo de testes em que participaram 54 pessoas entre 20 e 40 anos que pertencem ao programa social do ensino médio acelerado e foi aplicado o histórico clínico de detecção para determinar se estavam dentro dos critérios de inclusão. Aos 54 pacientes que preencheram o requisito, além do exame visual completo, foi aplicado o teste de Farnsworth, que é o teste mais preciso e completo para o diagnóstico das diferentes alterações na visão de cores. Resultados. Ao realizar a tabulação dos dados, observa-se que a alteração cromática mais frequente é a deuteranomalia, e que o agente mais neurotóxico para as células fotorreceptoras da retina é o álcool, afetando principalmente a população masculina entre 31 e 35 anos. 0 segundo neurotóxico mais agressivo é cigarros, afetando a mesma população. Conclusões. É determinado que os agentes neurotóxicos afetam diretamente o dano dos fotorreceptores especializados à cor, o dano pode ser imperceptível a princípio, mas pode afetar o paciente principalmente no desenvolvimento do trabalho, que é quando o paciente percebe que está apresentando uma alteração cromática, diminuindo a exposição a eles pode reverter o dano se a frequência não for prolongada.

Palavras-chave: Deuteranomalia; Farnsworth; fotorreceptor; neurotóxico; retina

\section{INTRODUCCIÓN}

$\mathrm{E}$ 1 motivo de la investigación fue determinar las alteraciones cromáticas por agentes neurotóxicos a través del test de Farnsworth en personas de edades entre 20 a 30 del bachillerato acelerado en la unidad educativa de la parroquia Checa de la Provincia de ichincha en Ecuador. Se demostró la importancia de la obtención de datos en una consulta optométrica mediante la anamnesis y la efectividad de la prueba de Farnsworth sobre otros test. Existe un porcentaje de Protanomalía en los pacientes y este puede deberse al consumo de tabaco y alcohol. El porcentaje de deuteranomalía detectado puede ser causado mayormente por el consumo exagerado de alcohol.

Desde la integración de los medios refringentes y la influencia del metabolismo propio de cada medio, metabolismo que los mantiene transparentes y funcionales, el mecanismo de acomodación, entre otros, hasta la disposición de las túnicas, todo, ha hecho que el ojo despliegue un sin número de funciones que, junto con la conexión al cerebro, permite que el ser humano desarrolle el sentido de la visión.

La conexión de todas las partes anatómicas del globo ocular con el cerebro, transforma a este órgano en un sentido sensorial, permitiendo al ser humano distinguir formas, distancias y la percepción de todo el espectro de colores, generando así, la visión cromática.

¿Dónde específicamente se perciben los colores? se perciben en la retina nerviosa. "En el centro de la parte posterior del ojo hay una zona de cerca de un milímetro de diámetro, llamada fóvea. Sólo contiene conos. La luz que llega a este pequeño sector proporciona la definición más precisa de color". (1).

La visión cromática se da gracias a las células fotosensibles de la retina que son los conos y los bastones. Cuando examinamos los detalles de una imagen, desplazamos nuestros ojos automáticamente hasta que lo que deseamos ver está encerrado en la fóvea. (1). 
Contemplamos los colores con mayor precisión en un campo muy angosto de la visión, sólo alrededor de unos 2 grados directamente ante nuestros ojos de los 360 grados del campo que nos rodea. (1).

Estas células especializadas de la retina, los conos, son capaces de ser sensibles a 3 longitudes de onda de colores primarios, conos L (longitud larga, sensibles al color rojo), conos M (longitud media, sensibles al color verde), y conos $\mathrm{S}$ (longitud corta, sensibles al color azul).

La percepción de los colores por el ser humano nunca es algo "absoluto", ya que la misma persona puede percibirlos diferente por distintos factores ya sean físicos o psíquicos que influyen en la percepción del color. (2).

También es importante saber que entre una persona a otra ven los colores de forma distinta. El único órgano capaz de percibir las radiaciones que llamamos color es "el ojo" junto con la retina y sus células (conos y bastones) (2).

Como es descrito por muchos autores, el color en sí mismo, no es una propiedad de la materia sino más bien el resultado de su interacción con la luz y a su vez de esta con el observador, dicho de otra manera, el color es una sensación que depende de tres factores: la longitud de onda de la luz, la capacidad de un objeto de reflejar o absorber dicha longitud de onda, y la capacidad de un observador de absorber dicho espectro luminoso convertirlo en una señal electromagnética que pueda ser interpretado por el cerebro para que este a su vez de respuesta a dicho estimulo. (3).

La visión cromática puede presentar algunas alteraciones por ciertos factores influyentes, estos pueden ser congénitos o adquiridos; quienes presenten cualquier tipo de alteración cromática tienen una imposibilidad o incapacidad de percibir algunas gamas de colores.

Las deficiencias cromáticas congénitas, suelen estar causadas por la ausencia o fallo en los fotopigmentos, que se manifiestas de diferentes maneras: desde la alteración de uno de los fotopigmentos y tener los otros normales (tricromátismo anómalo); la presencia de solamente dos tipos de fotopigmentos (dicrómata) o solamente uno o ninguno de ellos (monocrómata). Las deficiencias cromáticas adquiridas pueden afectar de la misma manera a hombres y mujeres (CITA); éstas pueden ser monoculares y binoculares. Puede deberse a enfermedades de tipo infeccioso (sífilis) o no infeccioso, como pueden ser las cataratas, el glaucoma o la degeneración macular, así como también por neurotóxicos que inhiben directamente el sistema nervioso central. (2).

Dentro de este estudio se hace hincapié en la Neurotoxicidad y su influencia directa en las alteraciones de la visión del color. La retina es una estructura $100 \%$ nerviosa y está conectada al cerebro directamente a través de las células ganglionares, al inicio se había mencionado que cada estructura del globo ocular tiene su propio metabolismo, es decir, todo lo que el ser humano ingiere o todo a lo que esté expuesto, afectará directamente el metabolismo de las estructuras, sea para nutrirlo y mejorarlo, o en el caso de agentes bilógicos, químicos $\mathrm{y}$ hasta físicos, que pueden influir directamente en el sistema nervioso, afectando directamente retina nerviosa degenerando en una neurotoxicidad, desencadenando así, una serie de insuficiencias en la visión del color.

Pero ¿Cuáles son los agentes ambientales que vuelven a la retina más 
vulnerable a una neurotoxicidad?, dentro de estos factores está la ingesta prolongada de fármacos, ingesta de sustancias psicoactivas y estupefacientes, exposición ocupacional a químicos o radiaciones e incluso el consumo de agua tóxica mezclada con químicos o respirar un aire viciado de igual forma, por químicos.

Los agentes neurotóxicos son aquellas alteraciones funcionales, estructurales, y bioquímicas que se producen en el sistema nervioso central lo que conlleva a la manifestación de varios efectos adversos como consecuencia de la exposición a un agente químico. El efecto adverso implica una alteración del sistema nervioso central, esta puede ser neuroquímica, morfológica o puede estar relacionada con la conducta, y puede manifestarse transitoria o permanente. La mayoría de los agentes neurotóxicos afectan al sistema nervioso central como periférico, los que afectan a este último son más fáciles de reconocer ya que los síntomas observados son más específicos. (4).

Dentro de una consulta optométrica para poder determinar si un paciente presenta alteración cromática se sigue el mismo protocolo de un examen visual, pero es de suma importancia realizar una buena anamnesis, y se debe prestar atención al motivo de consulta, los pacientes pueden mencionar que se realizaron la prueba de conducción y fue entonces que se dieron cuenta que fallaba su visión de color, o que compararon la percepción de un color en relación a un grupo de personas y mencionar que se dieron cuenta que su percepción era distinta a la de la mayoría.

Si el motivo de consulta es parecido a lo mencionado con anterioridad, hay que ser minucioso al momento de recabar datos con preguntas orientadas a la exposición de agentes químicos, aire tóxico, consumo de medicamentos $\mathrm{y}$ sustancias psicoactivas y los tiempos de consumo o exposición a los mismos. Posterior a la aplicación del protocolo de un examen visual, el test más confiable para valorar alteraciones de la visión del color es el test de Farnsworth.

El test está compuesto por 16 pastillas de colores escogidas en atlas de Munsell de forma que los intervalos entre tonos sean aproximadamente iguales o similares, así como la luminosidad y la saturación: las pastillas de colores están numeradas del 115 en la parte posterior y una "P" para la pastilla referencia. Dado que las fichas de color tienen la misma luminancia para la observación normal, la discriminación entre fichas se basa únicamente en el color. (5).

Los resultados de Farnsworth son precisos, se aplica en casos como: pacientes con sospecha o con inflamación del nervio óptico, pacientes expuestos a químicos, neurotóxicos y medicamentos, pacientes que han presentado alteración cromática con las cartillas del test de Ishihara, pacientes que consumen cloroquina y otras medicaciones que pueden causar toxicidad a nivel de macula.

Es así que la investigación tiene como finalidad descubrir si los agentes neurotóxicos a los que una población, de entre 20 a 40 años, que los consuma o a los que esté expuesto, son potenciales desencadenantes de una deficiencia o alteración cromática a través de la valoración de test que ayudan a identificar fallos en la percepción del color. 


\section{MATERIALES Y MÉTODOS}

$\mathrm{P}$ ara obtener los resultados necesarios de este estudio, se aplicó el test de Farnsworth a 54 personas comprendidas en edades de 20 a 40 años, la muestra se obtuvo utilizando el primer instrumento que fue una encuesta con preguntas cerradas relacionadas a si los pacientes consumían alcohol, cigarrillo $\mathrm{u}$ otros estupefacientes considerados como neurotóxicos, a los pacientes cuyas respuestas fueron positivas, se les sometió a un examen visual para aplicar el segundo instrumento que fue la historia clínica.

Se realizó un examen visual completo que incluyó la prueba de visión del color con el test de Farnsworth. Se procesaron los resultados de la historia clínica, y se realizó el análisis principalmente de la prueba cromática, para el procesamiento de este test se basó específicamente en la guía de resultados que proporciona la misma guía de esta prueba $y$, según la respuesta de cada paciente la fiabilidad del resultado está entre 80 a 95\%.

\section{RESULTADOS}

S e obtuvo resultados de los 54 pacientes que participaron en la investigación, en la que 59\% fueron de género masculino (Tabla 1), ya que el lugar en el que se obtuvo la muestra es un establecimiento de bachillerato acelerado $\mathrm{y}$ ducho género tiene mayor demanda de estudio.

Tabla 1. Género

\begin{tabular}{ccc}
\hline GÉNERO & NÚMERO & PORCENTAJE \\
\hline HOMBRES & 32 & $59 \%$ \\
MUJERES & 22 & $41 \%$ \\
\hline
\end{tabular}

Además, el 39\% de los pacientes se encontraron en un rango de edad de 31 a 35 años, puesto que es una edad económicamente activa y, la exigencia de educación para conseguir trabajo en el país es alta, las edades con demanda para obtener educación es la obtenida en los resultados, como se evidencia en la Tabla 2.

Tabla 2. Rango de edad

\begin{tabular}{ccc}
\hline RANGO & NÚMERO & PORCENTAJE \\
\hline $20-25$ AÑOS & 15 & $27 \%$ \\
$26-30$ AÑOS & 10 & $19 \%$ \\
$31-35$ AÑOS & 21 & $39 \%$ \\
$36-40$ AÑOS & 8 & $15 \%$ \\
\hline
\end{tabular}

Para los fines de estudió se tabularon los rangos de agudeza visual y defectos refractivos es así que, para agudeza visual, se encontró un rango de AV 20/20-25 del 59\% (Tabla 3), que indica que más de la mitad de los pacientes, no es completamente libre de deficiencias visuales pero que a su vez los defectos no serían altos. 
Tabla 3. Agudeza visual

\begin{tabular}{ccc}
\hline RANGO DE AV & PACIENTES & PORCENTAJE \\
\hline $20 / 20-20 / 25$ & 32 & $59 \%$ \\
$20 / 25-20 / 40$ & 12 & $26 \%$ \\
$20 / 40-20 / 60$ & 8 & $15 \%$ \\
\hline
\end{tabular}

Sobre deficiencias visuales propiamente dichas la mayor cantidad de pacientes fueron miopes en ojo derecho: $38 \%$, ojo izquierdo: $42 \%$, seguido de emétropes ojo derecho: $22 \%$, ojo izquierdo: $18 \%$, y, por último, astigmatismo miópico simple $15 \%$ en ambos ojos como se detalla en la Tabla 4. Esto podría ser un indicativo de asociación de una determinada alteración cromática con un determinado defecto visual o que, a su vez, la presencia de determinado defecto visual, vuelva al paciente más vulnerable a adquirir una alteración cromática.

Tabla 4. Defectos visuales

\begin{tabular}{ccccc}
\hline & MIOPÍA & HIPERMETROPÍA & $\begin{array}{c}\text { ASTIGMATISMO } \\
\text { MIÓPICO SIMPLE }\end{array}$ & EMETROPES \\
\hline OD & $38 \%$ & $22 \%$ & $15 \%$ & $25 \%$ \\
OI & $42 \%$ & $18 \%$ & $15 \%$ & $25 \%$ \\
\hline
\end{tabular}

En los hallazgos de anomalías de la visión cromática propiamente dichas, de los resultados del test de Farnsworth se encuentra discromatopsias tritan en mayor porcentaje, siendo del $70 \%$ la presencia de esta alteración cromática, seguido de discromatopsias protan presente en $17 \%$ de la muestra y en un 13\% la presencia de las deuteranomalías que se evidencia en la Tabla 5. De estas discromatopsias se pudieron obtener resultados que neurotóxicos específicos desencadenan en mayor o menor grado dichas deficiencias del color.

Tabla 5. Frecuencia total de discromatopsia.

\begin{tabular}{ccc}
\hline VARIABLE & FRECUENCIA & PORCENTAJE \\
\hline PRONTA & 8 & $17 \%$ \\
TRITAN & 38 & $70 \%$ \\
DEUTAN & 7 & $13 \%$ \\
\hline TOTAL & 54 & $100 \%$ \\
\hline
\end{tabular}

Con los resultados del test de Farsnsworth y la anamnesis, se definió que el neurotóxico que mayor porcentaje de anomalías de la visión causó, pudo haber sido el alcohol, desencadenando $56 \%$ de protanomalías, $71 \%$ deuteranomalías y 29\% tritanomalías, el segundo neurotóxico fue el tabaco para las protanomalías (44\%), los químicos para las deuteranomalías (21\%) y el alcohol, las drogas y los químicos para las tritanomalías. Estos resultados se evidencian en las Tablas 6, 7 y 8, además, se observan otros agentes que causan discromatopsias, pero en menor porcentaje. 
Tabla 6. Cuadro de protanomalía por neurotóxicos.

\begin{tabular}{ccc}
\hline VARIABLE & FRECUENCIA & PORCENTAJE \\
\hline ALCOHOL & 5 & $56 \%$ \\
TABACO & 4 & $44 \%$ \\
DROGAS & - & \\
QUÍMICOS & - & \\
MEDICAMENTOS & - & \\
\hline TOTAL & $\mathbf{9}$ & $\mathbf{1 0 0 \%}$ \\
\hline
\end{tabular}

Tabla 7. Cuadro de deuteranomalía por neurotóxicos

\begin{tabular}{ccc}
\hline VARIABLE & FRECUENCIA & PORCENTAJE \\
\hline ALCOHOL & 27 & $71 \%$ \\
TABACO & 1 & $3 \%$ \\
DROGAS & - & $0 \%$ \\
QUÍMICOS & 8 & $21 \%$ \\
MEDICAMENTOS & 2 & 5 \\
\hline TOTAL & $\mathbf{3 8}$ & $\mathbf{1 0 0 \%}$ \\
\hline
\end{tabular}

Tabla 8. Cuadro de tritanomalía por neurotóxicos

\begin{tabular}{ccc}
\hline VARIABLE & FRECUENCIA & PORCENTAJE \\
\hline ALCOHOL & 2 & $29 \%$ \\
TABACO & - & $0 \%$ \\
DROGAS & 2 & $29 \%$ \\
QUÍMICOS & 2 & $29 \%$ \\
MEDICAMENTOS & 1 & 14 \\
\hline TOTAL & $\mathbf{7}$ & $\mathbf{1 0 0 \%}$ \\
\hline
\end{tabular}

\section{Discusión}

La presencia de discromatopsias tritan que se registra en la población estudiada se atribuye a la exposición de agentes neurotóxicos y el consumo de dichos agentes, en especial del alcohol. Es posible que esto traiga consecuencias mayores si la exposición es más frecuente, las células receptoras del color podrían mejorar la condición de alteración cromática si se elimina por completo la exposición a estos agentes tóxicos
El resultado de deuteranomalías, en un porcentaje mayor a de las tritanomalías y protanomalías, es similar a los encontrado en investigaciones previas hechas por la Universidad de La Salle y en contextos donde prevalece el consumo del alcohol siendo este, el agente neurotóxico más relevante en la presencia de las alteraciones del color, por tanto se corrobora que existe una completa asociación entre la influencia directa del consumo o la exposición de agentes neurotóxicos que no es nada contrario a lo que se plantea en la hipótesis. 
Los resultados de porcentajes altos encontrados en este estudió no son diferentes a estudios antiguos que se hicieron en otros países de Latinoamérica a pesar que dichos estudios tuvieron un tiempo de seguimiento mayor a este investigación que fue en un periodo más corto. Para este estudio la muestra fue menor a la indagada en otras investigaciones.

Los resultados obtenidos en este estudio son solo comparables por metodología con los realizados obtenidos en la investigación de la Universidad de la Salle por lo que existe la posibilidad que existan cambios temporales en las alteraciones ante la frecuencia de consumo según la población u otras variables como las edades o el género ya que si la muestra modifica estas variables, probablemente la predominancia de las discromatopsias puede cambiar y el agente neurotóxico influyente también.

Tanto por su relevancia teóricoconceptual como por sus implicaciones clínicas, debería profundizarse en la investigación del constructo puesto que dentro de las alteraciones cromáticas, la presencia de defectos visuales también puede generar vulnerabilidad en la pérdida de la percepción del color.

Se podría afirmar entonces, que los agentes neurotóxicos que son consumidos por la muestra tales como, el alcohol, el cigarrillo, tabaco y ciertos medicamentos son totalmente influyentes en el daño o alteración permanente o momentánea de las células receptoras del color que se encuentra en la retina. Todos los sujetos de estudio presentaron en mayor o menor grado una resultante de alteración cromática.

Teniendo en cuenta que el alcohol es un potente indicador del riesgo como neurotóxico para las células receptoras del color, los resultados obtenidos en este estudio pueden apuntar a que nivel de riesgo de discromatopsias es alta pero puede mejorar la condición de alteración del color si se elimina la exposición a los tóxicos.

\section{CONCLUSIONES}

$\mathrm{E}$ 1 estudio demuestra evidencia de que los agentes neurotóxicos pueden tener una influencia directa en las células receptoras del color. La población estudiada estuvo expuesta al alcohol, el cigarrillo, químicos y, en menos proporción, consumían prolongadamente medicamentos para el tratamiento de alguna enfermedad sistémica. De los pacientes con deuteranomalía, fueron pacientes masculinos que principalmente consumían alcohol. Las células fotorreceptoras podrían mejorar y hasta volver a restituirse, eliminando la alteración cromática, si se disminuye o se elimina por completo el consumo de estos agentes, la exposición a químicos o se elimina el medicamento que influya en la alteración de los conos. Se debería implementar programas de evaluación y prevención de esta clase de anomalías visuales con la coordinación de centros de salud locales y el Ministerio de Salud Pública de Ecuador. La falta de investigaciones en el país denota un amplio campo de estudio nuevo que podría fortalecerse con el desarrollo de proyectos de titulación en el área de optometría y oftalmología de las distintas instituciones educativas.

Ya que el motivo de la investigación fue determinar las alteraciones cromáticas por agentes neurotóxicos a través del test de Farnsworth, como se menciona en la 
introducción, se comprueba que los agentes neurotóxicos sí pueden causan alteraciones en las células nerviosas fotorreceptoras del color y la más frecuente por agentes neurotóxicos es la deuteranomalía.

Se determina que la alteración cromática adquirida por neurotóxicos dependerá del tiempo de exposición o consumo de los mismos.

Se considera que si la frecuencia de consumo o exposición de los neurotóxicos fue baja, y el paciente elimina el consumo o la exposición de agentes neurotóxicos, las células pueden recuperarse, ya que es una alteración secundaria adquirida y no una falla genética.

Se concluye que el test de Farnswhorth es el test más acertado para la detección cualitativa de la visión cromática y no debería faltar esta prueba en la consulta optométrica si el paciente durante la anamnesis expresó que consume alcohol, cigarrillos, está expuesto a químicos por su ocupación, o si consume prolongadamente algún tratamiento con medicamentos químicos. (6)

Finalmente se evidencia que la población más vulnerable fueron los hombres de edades de entre 31 a 35 años.

\section{REFERENCIAS BIBLIOGRÁFICAS}

1. Zelanski P, Fisher MP. Color. Ediciones AKAL. 2001; 48
2. García P, Guzmán J. Neurotoxicidad y discromatopsias adquiridas (julio 30 de 2008 ed.). 2008; (P. E. García Alvares, \& J. Guzmán Vargas, Edits.) Bogotá: Fundación Universitaria del área Andina. Recuperado el 30 de Julio de 2008

3. Bolaños Godoy DA. Evaluación de la visión cromática en trabajadores de la industria textil expuestos a solventes orgánicos. 2018

4. Regidor Braojos L, Solans Lampurlanès $X$. Centro nacional de condiciones de trabajo. (27 de agosto de 2010). Obtenido de Centro 104 Nacional De Condiciones De Trabajo:http://www.insht.es/InshtWeb/C ontenidos/Documentacion/FichasTecnicas /NTP/ Ficheros/401a500/ntp_487.pdf. 2010.

5. Rodríguez Pinzón DC, y Orjuela Rozo A. Determinación de alteraciones cromáticas. [Tesis]. Bogotá, Colombia. 2008. Obtenido de

http://repository.lasalle.edu.co/bitstream /handle/10185/8573/T50.08\%20R618d. $\mathrm{p}$ df? sequence $=1$ \&isAllowed $=y$

6. Chaparro Morales I. Aplicación de la prueba Farnsworth Hue 100 en trabajadores expuestos a pesticidas, para detección de alteraciones adquiridas al color. 2018

Conflicto de intereses. Las autoras declaran que no existe conflicto de intereses para la publicación del presente artículo.

Financiamiento. Autofinanciamiento

Agradecimiento. No declaran 